\title{
Defining a conceptual framework for the integration of modelling and advanced imaging for improving the reliability and efficiency of bridge assessments
}

\author{
Brodie Chan ${ }^{1,2 \mathrm{a}}$, Hong Guan ${ }^{1 *}$, Lei Hou ${ }^{1 \mathrm{~b}}$, Jun Jo ${ }^{3 \mathrm{c}}$, Michael Blumenstein ${ }^{4 \mathrm{~d}}$ and Jun Wang ${ }^{5 \mathrm{e}}$ \\ ${ }^{1}$ Griffith School of Engineering, Griffith University, QLD 4222, Australia \\ ${ }^{2}$ GHD Pty Ltd, Brisbane 4000, QLD, Australia \\ ${ }^{3}$ School of Information and Communication Technology, Griffith University, QLD 4222, Australia \\ ${ }^{4}$ School of Software, University of Technology Sydney, NSW 2007, Australia \\ ${ }^{5}$ Australasian Joint Research Centre for Building Information Modelling, Curtin University, WA 6102, Australia
}

\begin{abstract}
Current bridge inspection practices are typically predicated upon manual paper-based data collection methods, which significantly limit the ability to transfer knowledge gained throughout the lifecycle of the asset, to benefit the assessment of the inspector or engineer. This study aims to overcome the limitations of current practices and proposes a conceptual framework to improve the reliability and efficiency of current bridge asset management practices through the integration of Building Information Modeling (BIM) and advanced computing and imaging technologies. As a tool for bridge inspections, BIM offers significant potential when integrated with laser scanning and keypoint-based texture recognition, which allows for the detection of such defects as cracking, corrosion or settlement in bridge components. In recent years, the construction industry has seen an increased use of BIM technology on site to aid the construction process. However, the applications of it are deficient through the asset management phases of a project. Given the ability of BIM to house all component specific information gathered from the construction, inspection and maintenance phases, BIM is envisioned to allow emphasis to be placed on retrieving the relevant information throughout the project lifecycle, ultimately enabling engineers and bridge inspectors to make more informed decisions about the current condition of the structure. Using BIM as the focal point for information collection throughout the project lifecycle, findings from advanced imaging and data processing are proposed to be stored within the model for recall at future bridge assessments.
\end{abstract}

Keywords: BIM, keypoint-based texture recognition, laser scanning, bridge inspection, condition assessment, bridge asset management

\footnotetext{
*Corresponding author, Professor, E-mail: h.guan@griffith.edu.au

aBEng, E-mail: brodie.chan@hotmail.com

${ }^{\mathrm{b}}$ Ph.D. E-mail: lei.hou@griffith.edu.au

${ }^{c}$ Ph.D. E-mail: j.jo@griffith.edu.au

${ }^{d}$ Ph.D. E-mail: Michael.Blumenstein@uts.edu.au

${ }^{\mathrm{e}} \mathrm{Ph} . D$. Candidate. E-mail: jun.wang15@postgrad.curtin.edu.au
} 


\section{Introduction}

Visual condition inspections remain paramount to assessing the current deterioration status of a bridge and assigning remediation or maintenance tasks so as to ensure the ongoing serviceability of the structure (Chan et al. 2015). Over the last few years, the restructuring of Australian economic climate in conjunction with limited government investment into new transport infrastructure has placed increasing importance upon these routine visual inspections. Throughout these inspections, bridge inspectors/engineers are to identify the condition of each individual bridge component in order to identify any repair/rehabilitation works required, and to forecast the future performance of the structure. This leads to a significant amount of bridge inspection data that is to be collected by inspectors, assessed by engineers and stored for future use (DiBernardo 2012). However, current visual inspection practices suffer a number of limitations: (i) human vision-based inspections are subjective and rely upon the inspector to accurately capture all information; (ii) the entire manual process is costly and time-consuming; (iii) a number of safety risks are associated with field inspectors; (iv) the inspection requires experienced and highly trained personnel and most bridge authorities are currently facing the issue of shortage of required level of qualified inspectors (Bu et al. 2012).

To exacerbate the current situation, asset owners largely utilise Bridge Asset Management Systems (BAMS) or Bridge Information Systems (BIS) to store and capture data that is collected from Level-2 condition inspections. However, as the name suggests, these asset management systems are typically centred on databases that provide no direct representation or visualisation of what the data means. In a study conducted by DiBernardo (2012), it was concluded that these database-oriented forms of BAMS lead to the issue of data dispersion, where key information collected in condition inspections can be obscured by the low efficiency of the systems. This can often prevent engineers from being able to fully understand how the condition of a structure has changed over time, which has the potential to negatively influence the decisions made relating to the repair, rehabilitation or remediation.

\section{Current inspection practices and systems}

The failure of the I-35W Bridge in August 2007 has highlighted the importance of ensuring consistency throughout inspections and the need for more assertive types of technologies to evaluate the current condition of bridge assets (Alampalli and Rehm 2011). From the report produced by the National Transportation Safety Board (2007) it was concluded that amongst other issues, there was a "lack of inspection guidance for conditions of gusset plate distortion and inadequate use of technologies for accurately assessing the condition of gusset plates. This report ultimately highlighted the need for alternative approaches to bridge inspections in order to more accurately monitor the current condition of key structural elements. However, this collapse is not only an isolated occurrence, with over 44 bridges in the US having failed between 1989 and 2000 as a result of poor maintenance and inspection practices (Wardhana and Hadipriono 2003). Extensive research has already been conducted into the use of destructive and non-destructive techniques, which are applicable to determining the in-situ condition of principal bridge components. This includes research conducted by Subhani et al. (2013), which provides an indication of the embedment length and deterioration for round timber elements, such as power poles or even piles. Methods of structural damage detection using vibration and other noise signals were outlined by Handara et al. (2012). Wang and Hao (2012) have investigated the use of vibration, guided wave and acoustic emissions data for the integrated monitoring of reinforced concrete beams. 
Additionally, a number of studies have been conducted into methods for predicting deterioration to a bridge and its principal components using probabilistic models $(\mathrm{Bu}$ et al. 2012; Lee et al. 2011).

The current approach to bridge asset management within Australia largely follows a cyclic process, whereby the findings of a condition inspection govern the successor activities, which include the designation of maintenance or repair activities and even the timing of subsequent inspections. This approach to bridge inspections places a high importance on accurately assessing the current condition of individual components, as failure to identify defects or deterioration mechanisms can lead to inadequate maintenance and repair actions. Within Australia, data capture on site is largely centred on paper-based inspection methods, whereby a condition rating is assigned to all components and all defects are captured and presented as a report which is issued to the client. This information typically feeds into an overall BAMS or database that is used to identify issues of high criticality and assign work orders accordingly. However, the key issue with this approach is that it is typically associated with a more reactive approach to asset management since the focus is placed upon addressing rectifying the condition of defective components. In a study by Bu et al. (2014b), it was noted that current decisions on maintenance, repair and rehabilitation are based on interpretations of information from databases about the structure as a whole. However, to ensure the effectiveness of activities, engineers require a deeper understanding of the individual defects present from construction, an understanding of how that particular component has deteriorated, previous repairs that have been undertaken for that element and the effectiveness of those repairs.

Defects that occur in one bridge element and not the others of the same element type may not necessarily be due to the deterioration. This can be attributed to a number of issues, including construction defects, different loading conditions (due to relative location) and etc. However, using current paper-based processes, the condition of similar types of elements are grouped together when reported on. Taking the example of bridge deck units, all of the units within a given span are grouped together as a single line item, with the number of deck units in each condition state highlighted. While this provides a high-level understanding of the defects required to be addressed, it fails to capture the condition of each individual deck unit and can mask the underlying cause of the defect. As highlighted by previous studies conducted by DiBernardo (2012), current database-oriented BAMS create subjective assessments that significantly affect decision-making. This is due to the discrete nature of current systems, whereby the information from a variety of sources feeds into the overall database but is not looked at collectively to provide an indication of the current condition. Ultimately, remedial actions assigned may not necessarily provide the optimal solution that accounts for deterioration mechanisms and balances serviceability and cost-effectiveness, but will have a bias towards one as opposed to the other. This is further illustrated through Fig. 1, with the typical systems demonstrated. 


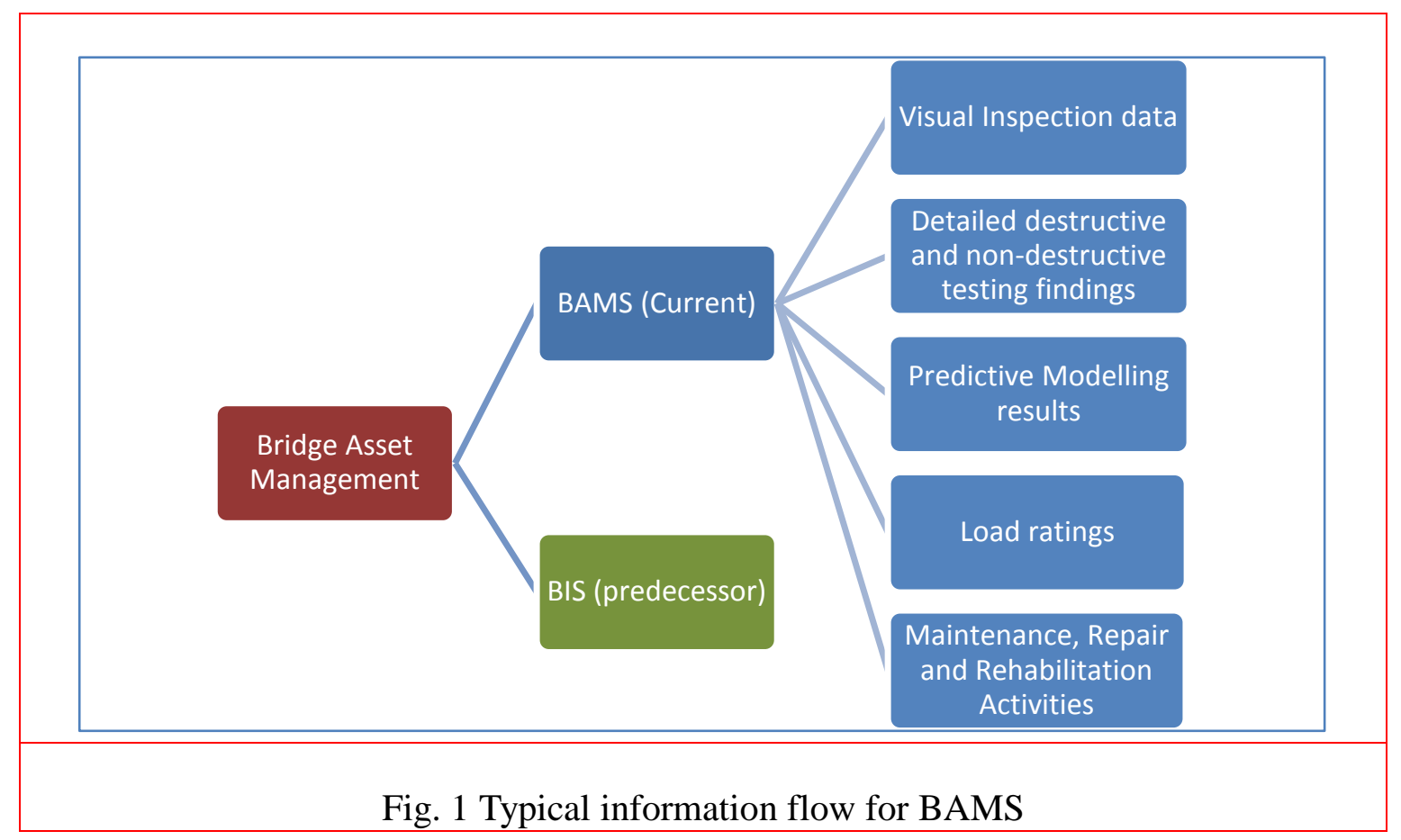

With recent strides forward in advanced imaging processes and 3-D modelling, Building Information Modelling (BIM) and keypoint-based texture recognition have been presented as prospective processes that are applicable to asset management. BIM is a technology that can be applied in numerous fields, such as construction management, facility operation and structure and Mechanical Electrical and Plumbing (MEP) design. BIM enables the representation of digital building information at any construction stages. For bridge infrastructure, BIM possesses the potential to integrate information relating to condition states and deterioration with the individual components, which traditional 2-D modelling systems were constrained by. However, more importantly, it is recognised that BIM enables better visualisation of the condition of an asset and greater interoperability (Marzouk and Abdelaty 2012). This ultimately enables inspectors to overcome previous limitations of paper-driven processes to deliver more informed decisions on the current condition and future maintenance requirements of a bridge. While it is often noted that BIM is capable of being used throughout the lifecycle of a project, the development and research conducted into the implementation of BIM for asset management has been limited (Riu and Issa 2016). This view is supported by Becerik-Gerber et al. (2012) as there is insufficient empirical data on the topic. A study conducted by McGuire et al. (2016) investigated the use of BIM to facilitate bridge evaluations and assessments by recording the location and extent of damage in the 3-D model. This information was collected and assigned directly to the model by placing 'damage cubes' onto a given element to represent volume and severity. Within this research, it was highlighted the ability of BIM to maintain parametric information about the bridge and visually represent damage, for which could be used in damage evaluations and load ratings. However, a gap currently exists in the application of BIM for field-based data collection and the understanding of how this could be uniformly applied to inspect and manage bridge infrastructure. This paper thus defines a conceptual framework for the implementation of BIM for in-the-field data collection through the integration with advanced imaging techniques. This integration between the two technologies is intended to overcome current limitations and provide more reliable means of collecting visual inspection data and housing information from destructive and non-destructive test methods. It must be noted that BIM is not to replace current methods of detailed testing and those outlined in the research above, 
but is intended to bring all data and information together into a model that acts as a single point of truth across the life of an asset.

\section{Conceptual framework}

As outlined previously, a key hindrance to regular condition assessments is the subjective nature of the inspection since they are reliant upon the experience and training of the inspector/engineer (Kamya 2010). A common contention arising throughout condition inspections is the criticality of a defect. For example, a crack may be seen as being moderated by one inspector, but may actually be due to fretting of the edges of the crack at the point of measurement. To overcome these limitations and realise more effective asset management practices, state-of-the-art technologies for crack and defect segmentation and detection in bridge images have been considered in this study.

\subsection{Pilot study methodology for keypoint-based crack detection}

The Scale-Invariant Feature Transform (SIFT) has been applied to many areas such as object or face detection, mapping and navigation, gesture recognition, etc. (Huang et al. 2008). This algorithm has widely been used in computer vision to detect and describe local features in images using the concept of key point extraction, presenting significant potential for crack and defect identification in bridge inspections. SIFT can identify objects even among clutter and under partial occlusion, because the SIFT feature descriptor is invariant to image translation, scaling, and rotation, partially invariant to illumination changes and robust to local geometric distortion. Using the SIFT algorithm, the following process was devised to detect cracks:

(1) A camera mounted to an Unmanned Arial Vehicle (UAV) with a pre-programmed flight route takes images of a bridge surface, and gathers a density and image overlap that is sufficient to produce a photogrammetric model. A bridge inspector/engineer analyses the image and visually identifies areas of concern for the image processing technique to highlight regions of interest for later comparison. The inspector is needed only for the first inspection or when the system requests it.

(2) A pre-processing step is undertaken to enhance the bridge image quality and remove the noise from the input images, allowing for a more effective extraction of key features. The RGB colour space can also be converted to a device independent colour space that separates colour from intensity.

(3) Following pre-processing, the key features of each image are extracted and stored in a database in the format of feature vector (or keypoints). Storing features instead of all the pixels of each image significantly reduces the storage space and reduces computational complexity.

(4) The learning strategy is then applied to model crack detection and segmentation in bridge images.

(5) The model created is finally used to detect the cracks in the newly collected bridge images. 
(6) When the inspection is taken next time, the camera, with the support of a UAV and the SIFT algorithm, captures a new image of the exact size, angle and illumination. The feature set of the new image are to be compared with its old version that was stored in the database, and any new information represented in the image, which did not exist in the earlier version, can be identified.

This methodology has been applied to a set of old and new images for a reinforced concrete element whereby cracking was induced in the member for the purpose of this study. The keypoint-based texture recognition algorithm was applied to the two images taken of the same element, one with the crack and one without the crack. The successive feature keypoints were created by the algorithm and are marked in a number of different colours. As illustrated in Fig. 2(a) is the original image of the element, which has keypoints designated across the image. Comparatively, Fig. 2(b) contains a new feature, a crack, which was not a part of Fig. 2(a). As illustrated, this change generates a significant number of additional keypoints within the area of apparent cracking and requests the input of the engineer to confirm whether the additional keypoints are related to defects.

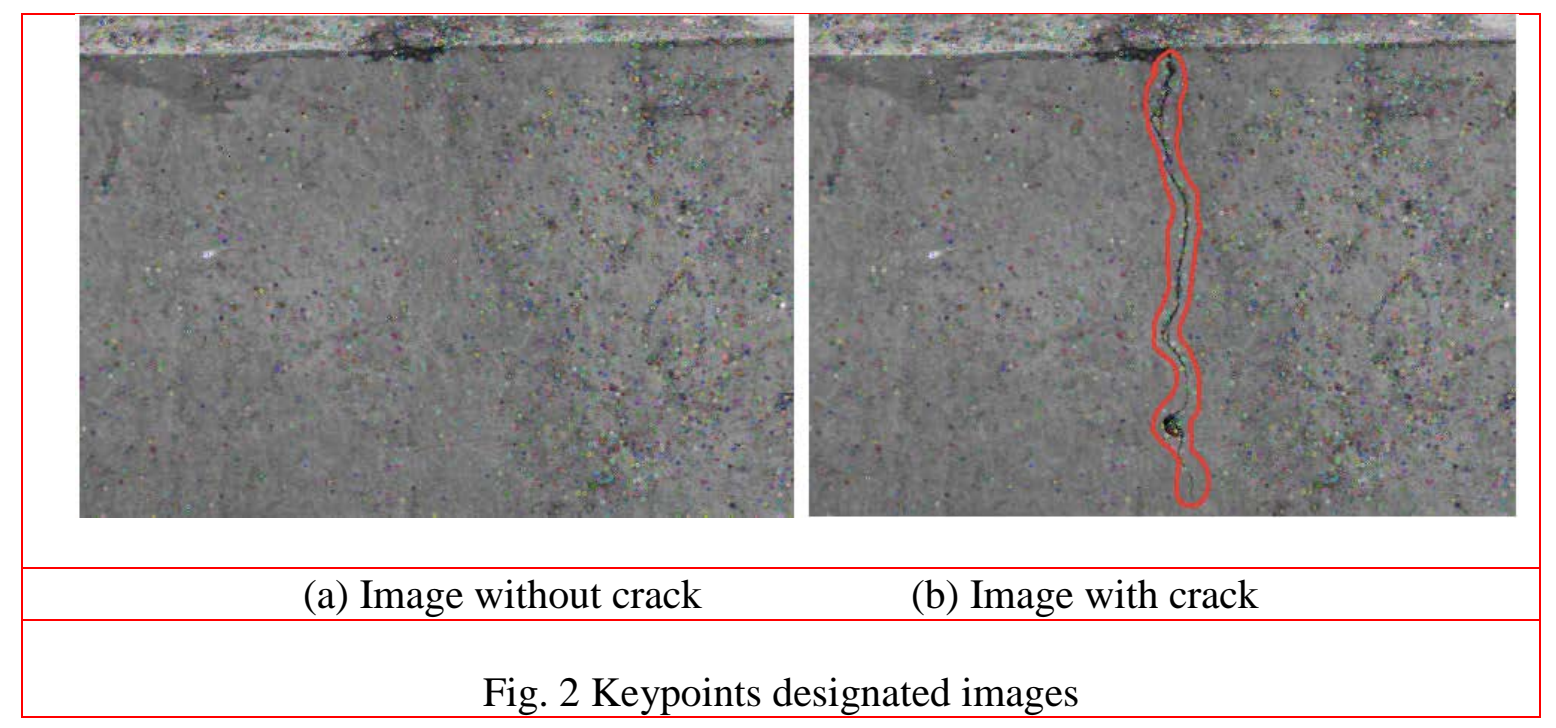

\subsection{Further system development for autonomous crack detection}

Like any classification method, the three steps, i.e. pre-processing, feature extraction and learning are considered imperative for obtaining crack types for each detected crack. Through applying different machine learning algorithms (Figueiredo et al. 2011) and image classification techniques (Adhikari et al. 2014), the system is able to replace the need for input from the human investigator and autonomously detects cracks. The machine learning algorithm for image registration will allow the system to address the limitations of randomness in viewing angles of the camera. Secondly, the image classification technique is able to identify the crack type and also demonstrate the difference between defects (i.e. cracking and spalling). Apart from this, an intelligent gimbal system will be developed, which can accurately guide the position and angle of the camera, considering information from multiple sensors (including Lidar, IMU and Ultrasonics where applicable). The high precision gimbal system and camera are to be mounted to a UAV. As a result, the UAV will be able to cover the structure and use the feature extraction and matching algorithm to identify defects and compare them to an existing image that is stored, where present, without the need for preprogrammed image capture locations. 


\subsection{Proposed BIM and keypoint-oriented bridge asset management}

Information such as cut-off levels, concrete mix designs, quality assurance documentation and other details should be assigned to individual bridge components to fulfill the greatest potential of BIM throughout the project lifecycle. Suites such as VEO M-Six, BIM 360 Field/Glue and Tekla Field 3D (AEC Magazine 2014) have presented as packages that have been marketed as being oriented towards asset management. These packages have the ability to input user-defined attributes and typically are able to collect information during bridge inspections, such as pictures, field notes, sketches, measurements and other information. Besides, Trimble Robotic Total Station and Layout Manager should be used to interface with the BIM model and input accurate cut-off levels and survey points (Trimble Navigation Ltd 2016). By housing all of the as-built information in a BIM model, this effectively ensures that the model can function as a true 4-D asset management model. With current manual inspection processes being subjective in nature, there is the need for BIM to be coupled with advanced imaging techniques, such as keypoint-based texture recognition. From images taken on site, algorithms such as SIFT, which have been developed for extracting distinctive features from images, could be used to identify such defects as cracking, spalling or corrosion staining detection. By matching and classifying individual features of an image and comparing it with the previous version taken (i.e. from a previous inspection), any features or defects that were not present in a previous image can be identified and quantified for comparison with keypoint images captured in subsequent inspections. As outlined previously, keypoint-based texture recognition has been shown to provide robust matching capabilities across a substantial range of affine distortion, change in 3-D viewpoint, addition of noise and changes in the illumination (Bu et al. 2015). This ultimately has a two-fold effect: reducing the subjective nature of condition inspections, whilst providing a consistent baseline from which future comparisons may be drawn to evaluate the extent of deterioration for the structure. These keypoint-based images are ultimately assigned to the individual elements of the structure for comparison at future inspections, allowing engineers to compare areas of previous concern and assess the extent of deterioration, all the while, housing the information in a semantic information-enriched 3-D model.

\section{Capacity of BIM-oriented BAMS}

It was highlighted by Taylor (2013) that the true value of BIM is its capacity to simplify the overall project lifecycle and ensure that information that is gained throughout each distinct phase (ie. from concept design through to operations/maintenance) is maintained. This is exemplified by the success of the Crossrail project in the United Kingdom (Crossrail Ltd 2015), whereby BIM has been utilised from the initial conceptual design, all the way through to the facilities management phase. Fig. 3 illustrates the three key stages of a project lifecycle and the intended integration of BIM throughout. With this in mind, the role of BIM for the asset management phase of a bridge lifecycle is to combine semantic information collected throughout the design and construction phases with information from inspections, detailed testing, monitoring, maintenance and most-significantly, advanced imaging techniques. The concept of BIM for bridge asset management is to break down the silos of current information storage processes and enable all information to be easily accessed and interrogated. 


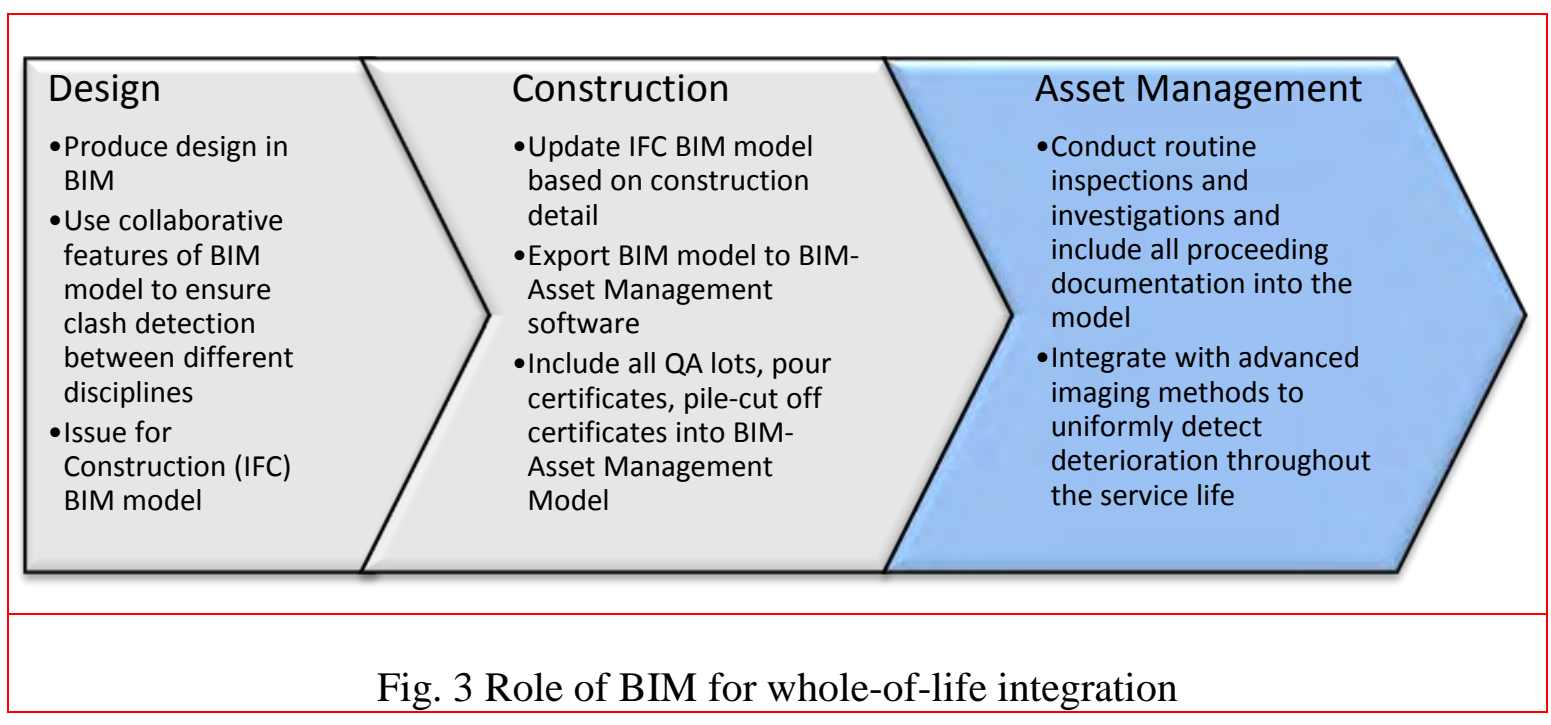

\subsection{Collection Efficiency of BIM}

With over 95\% of the costs of a project estimated as being spent over the operations and maintenance phase, there is sufficient rationale for improving current asset management processes (Hardin and McCool 2015). The most significant issue with the current BAMS and hence, means of data capture, is the inefficiency that is generated by the need to record recurring information for each individual bridge component. While condition inspections are typically conducted in a repetitive manner for a given structure throughout the design life, the need to recollect information that is already known increases the time required on site. The ability of BIM to model a structure using object-based elements, means that parametric information is able to be assigned to individual components, including photos, defect descriptions, construction details and other details about a bridge's condition (McGuire et al. 2016). As outlined by DiBernardo (2012), the 3-D model is able to integrate the data for each member with the information collected from field inspections, reducing the time required on site and increasing the efficiency of data collection.

\subsection{Flexibility of modelling based data collection}

One of the key benefits of housing/collecting information using modelling based techniques is that there remains the ability to output raw data (ie. Excel-based information). This remains a key strength of BIM, particularly in the transition towards more advanced systems as there is the ability to utilise new data capture means, whilst still outputting data that is compatible with current BAMS. Current BIM suites offer the ability to run queries on existing information, such as material properties, dimensions, construction detailing and any other information that is housed in the model (Marzouk and Hisham 2012). Hence, any inspection-related information that is collected and assigned to the model may be output in a format that is capable of aligning with current practices. 


\subsection{Depth of Condition Interpretation}

Fig. 4 illustrates the superstructure of a timber bridge pier that is modelled using Revit with condition state information assigned to the individual elements of the structure. As part of this, the components that are in a critical condition (with defects identified as affecting the integrity of the structure) are highlighted in red, with the elements that are 'as new' being shown in white. As demonstrated, much of the benefit of BIM lies in the ability to transfer knowledge of the locality and severity of defects to the users, with BIM assimilating semantic information such as condition state and component specific properties that contributes to decision making. Note that the conditions of bridge elements collected using the elementlevel bridge inspection process, are expressed quantitatively via the conventional "grading" system, i.e. the health index or the four condition states (with Condition 1 being "new" or "good”; Condition 2 "fair"; Condition 3 "poor"; and the Condition 4 "very poor"). Possessing this understanding enables a far more efficient and accurate analysis of the deterioration mechanisms of defects, which may be due to surrounding defects or loading conditions.

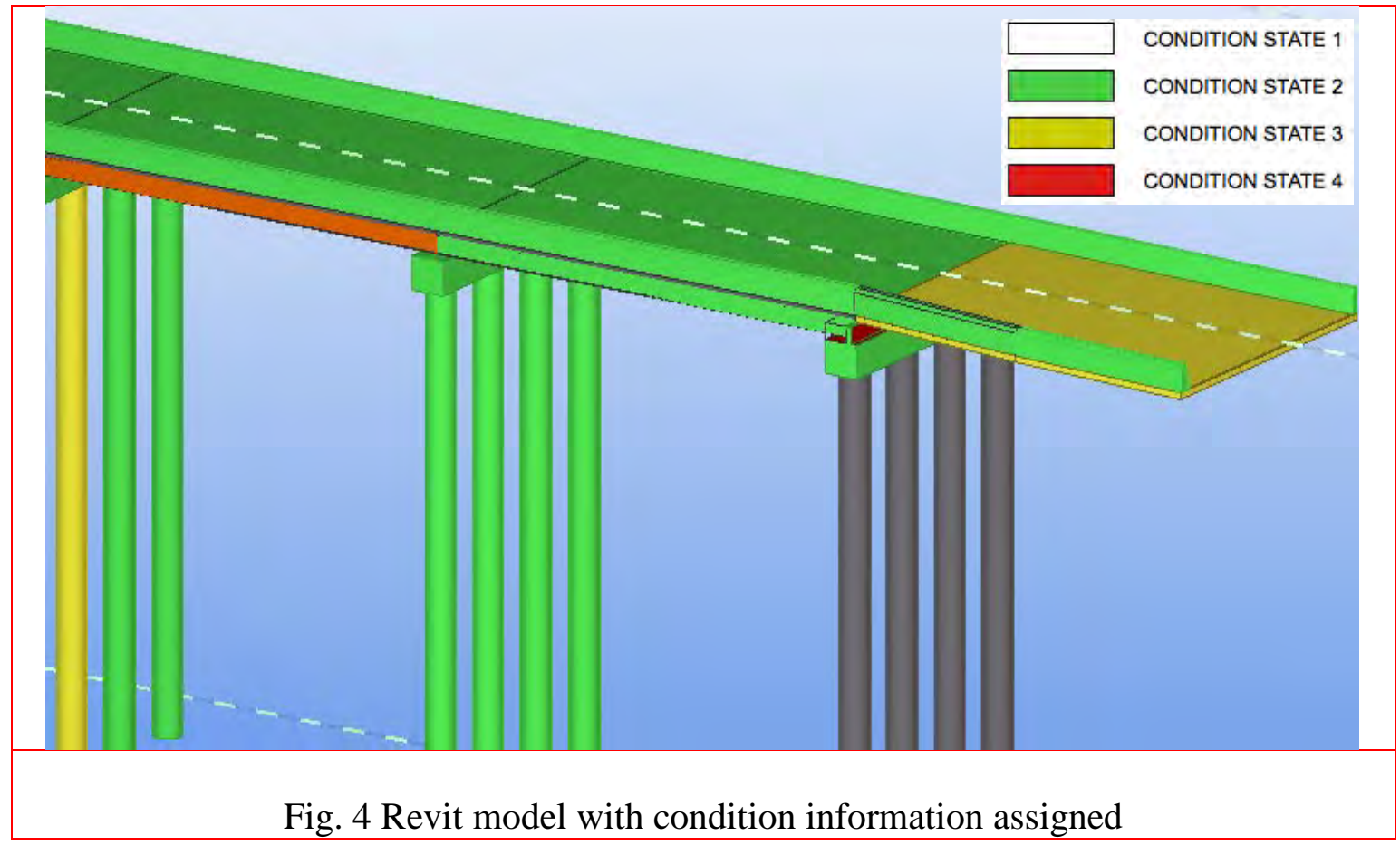

\section{BIM and Advanced Imaging Case Studies}

As an advanced and integrated design and construction solution, BIM in recent years has started to attain its universal acceptance in the building industry. It can be spotted from an overview of the global BIM applications that the majority of these applications still indwell the application level where collision detection and preliminary construction simulation are the primary undertakings prior to the construction stage. In order to rationalise the merits of $\mathrm{BIM}$ and the advanced imaging technologies across the project life cycle, especially in terms of providing immediate efficiencies and sweeping improvements on current asset/facility management processes, this section presents two case studies and concludes the lessons learned from the case studies. In the first case, the proposed solution for revitalising current practices included the use of BIM to provide accurate geometric modelling of the bridge components, in addition to housing any geometric data that was pertinent to a condition assessment. In the second case, a BIM plus 3-D laser scanning technology enabled inspectors 
to quickly and accurately obtain and visualise the spatial data of the project structure and identify the deviation from the comparison of the scan results and the as-planned BIM model. Through the use of BIM and laser scan it was envisaged that a single point of data storage,

\subsection{Pyrmont Bridge}

Being constructed between 1899 and 1902, the Pyrmont Bridge in Sydney, Australia forms a crucial link in the Darling Harbour network, connecting the city and inner western suburb. The structure spans $369 \mathrm{~m}$ and is comprised of 12 timber spans, with two steel central spans that form the 'swing spans' to allow vessels to pass through the channel. This bridge is now composed of more than 7300 structural members that are to be inspected and assessed by bridge engineers annually. With current processes being largely drive by manual data collection and entry, this became a timely and expensive process (Sahlman 2015). As a result, it was recognised by the current bridge management, the Sydney Harbour Foreshore Authority (SHFA), that there is the need to introduce more effective and efficient schemes to manage, inspect and maintain the structure. This included the intent to use digital processes, including electronic tablets, to provide greater efficiency and innovation to the current bridge asset management practice. As illustrated in Fig. 5, the model being represented in a proprietary asset management software, enables the visualisation of a select number of components, with the current condition rating of elements being represented by the colour array. The selected component shows previous photos taken and information that has been assigned, such as design drawings or previous work orders.

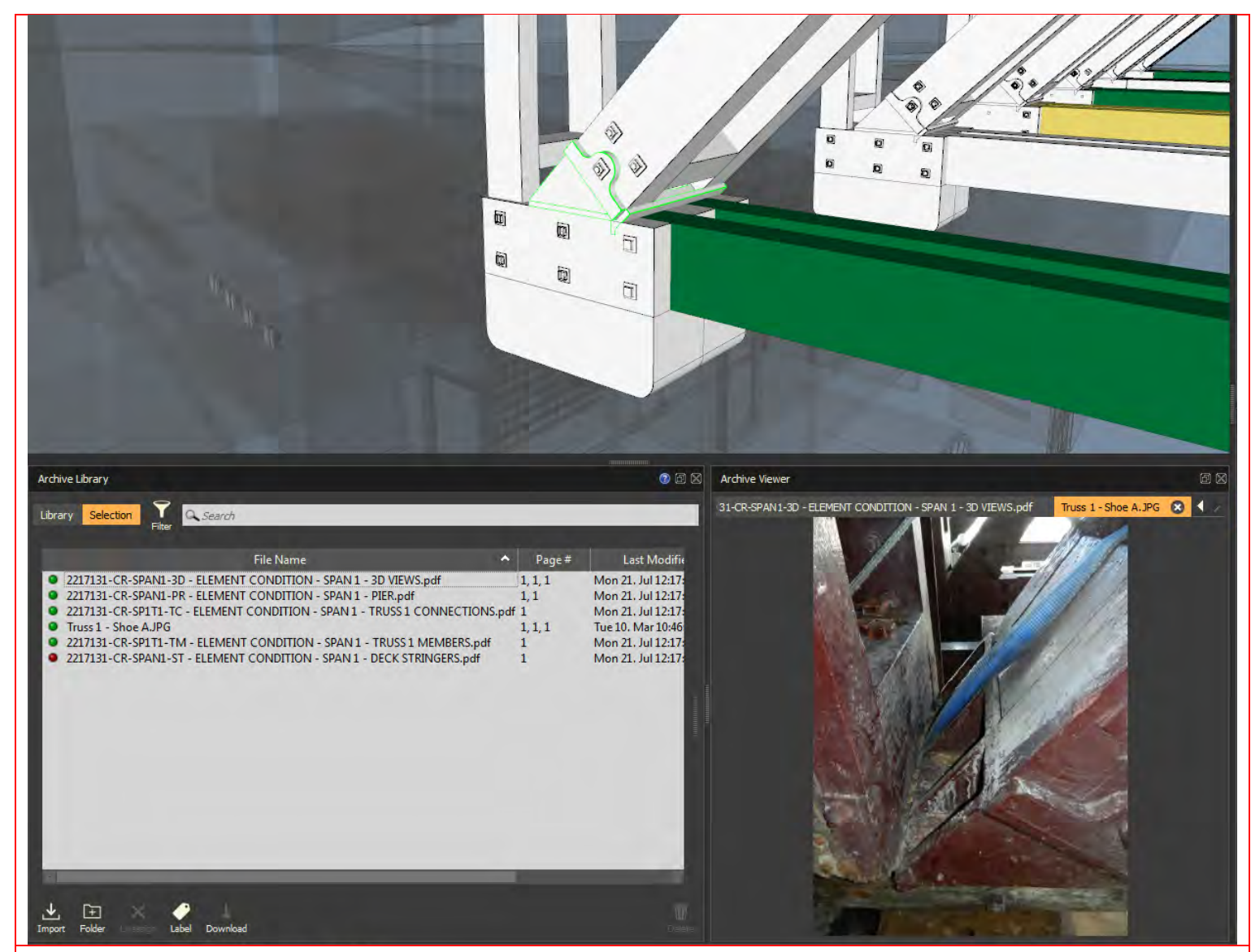

Fig. 5 Pyrmont Bridge truss shoe inspection (M-Six, 2015) 
Arguably, the most significant benefit to the bridge management was made through reducing the time required for all asset management processes. Inspectors are now able to link photos to the 3D model, assign commentary around the current condition and being able to identify the rate of deterioration for given components, such as the extent of rotting to timber truss members against previous inspections. Sequentially, information from the inspection is able to immediately be output to an inspection report through the query functions of BIM. In addition to this, removing the non-systemised manner of paper-based manual processes and moving towards modelling has driven a more proactive approach to asset management. From the condition inspections, the data is able to seamlessly be transferred into the maintenance and capital expenditure programs. The use of BIM has enabled the SHFA to more effectively prioritise maintenance activities, effortlessly determine the quantities for a given maintenance work order and document the maintenance history. For the Pyrmont Bridge, this has translated into considerable savings for producing maintenance work orders.

While the Pyrmont Bridge stands as an example of how BIM can improve asset management practices for complex structures, it also highlights the fine line between being useful and providing too much detail. While large levels of details such as the location and set out of nuts, bolts and packer plates is extremely valuable in the construction phase of a project, it can cloud the useful information when passed through to the asset management phase. Hence, from the start of the project, there is the need to define the minimum amount of information that is required to be included into a BIM model to ensure that the model is efficient, yet still useable. With such a large number of components, there is the additional constraint of having a large IFC export file size that influences the speed of the mobile capture software when in operation (Sahlman 2015). While the model can be optimised for viewing, there may still be the need to break this model down into manageable segments that align with the inspection workflow. For instance, the model may be separated into segments with the 10 timber approach spans being represented in a model that is separate to that for the steel swing spans.

The quality of an inspection is based upon the knowledge and experience of a bridge inspector and the ability to ensure that the findings of the inspection comply with the prescribed procedures of the relevant bridge inspection manuals. While inspectors are provided with the necessary tools to efficiently conduct inspections, there still exists the inherently subjective nature of condition assessments. For example, a crack in a reinforced concrete member is assigned a criticality by the maximum width of cracking. However, with current processes of crack determination being largely manual (i.e. using a crack gauge), an experienced inspector may deem that there is fretting at the surface of the crack opening which is not a true representation of the crack width. In the subsequent routine inspection, a less experienced inspector may not identify the fretting and deem that the crack has significantly increased from previous inspections. This will consequently flag up as an immediate concern to the asset owner, requiring immediate rectification.

\subsection{Yongxin Floodgate Pumping Station}

While the application of BIM throughout the asset management phase of bridge projects has been limited, there has been significant potential exemplified through the recent Yongxin Floodgate Pumping Station Project in China. The opportunity existing to introduce 3-D laser scanned modelling (i.e. generating point clouds) for the existing structure to allow engineers 
to more effectively and efficiently monitor that current rate of settlement and changes in the geographical features of the structure. This model could be compared to the existing as-built BIM model that had been produced, allowing for the tracking of deterioration and decay. The proposed approach to collect the existing terrestrial information was the use of 3-D laser scanning in order to produce a point cloud model. To collect the information, 3-D scanners were set at control locations around the site to allow for collection of different targets. A minimum of three control locations were required, which were set out appropriately in order to ensure the point cloud model could be accurately stitched together. As these laser scans from the different control locations are independent of each other, the point cloud data can be automatically combined based on feature points within each of the data sets to a singular coordinate system. For the floodgate pumping station, post processing was applied to remove any existing features that were not relevant to the model and the point clouds subsequently rendered to assign colours. The final point cloud model was imported into Bentley's Geopak software (Takken 2016), with the dense point cloud model producing a detailed digital terrain model.

From the Yongxin Floodgate Project, the key benefit was the ability to monitor the varying degrees of settlement for the flow channel, as surrounding ground of the flow channel. In order to explore the application of 3-D laser scanning approach in settlement observation and benchmark with the traditional electronic level gauging approach, the engineers conducted settlement monitoring at a daily basis using both approaches and laid a number of settlement monitoring points on the Larssen steel sheet around the foundation pits. Table 1 indicates the average measurement results derived from two different measuring approaches. The findings of the investigation indicated that both the 3-D laser scanning results and the levelling equipment outcomes reflected a same trend of settlement. There was up to a $7 \mathrm{~mm}$ variance between the two approaches, with an average variance equated to approximately $6.3 \%$.Based upon the analysis, it is witnessed that the accuracy of 3-D laser scanning can meet the requirements of settlement monitoring in the water conservancy project, however the efficiency of laser scanning may not be necessarily higher than the conventional electronic level gauge.

Table 1. Comparison of settlement results from two monitoring approaches

\begin{tabular}{|c|c|c|c|}
\hline $\begin{array}{c}\text { Date of } \\
\text { Measurement }\end{array}$ & $\begin{array}{c}\text { 3-D Laser } \\
\text { Scanner (mm) }\end{array}$ & $\begin{array}{c}\text { Electronic Level } \\
\text { Gauge (mm) }\end{array}$ & $\begin{array}{l}\text { Disparity } \\
\text { (mm) }\end{array}$ \\
\hline April 2 $2^{\text {nd }}, 2015$ & -190.5 & -197.5 & -7 \\
\hline April $25^{\text {th }}, 2015$ & -53 & -47.5 & 5.5 \\
\hline May $28^{\text {th }}, 2015$ & -128 & -121.8 & 6.2 \\
\hline
\end{tabular}

The water channel is a relatively crucial and complex part in this project. The engineers also used 3-D laser scanners to scan the entire flow channel layer, and evidenced that the relative accuracy of the model allowed for more efficient methods of monitoring the settlement of the structure and changes in the overall condition over time. Fig. 6 illustrates the point cloud model that is overlaid within the original BIM model, which highlights the differences in the surface profile. This is further illustrated by the cross-sections taken through the model, as shown in Fig. 7. These cross sections highlight the localised variations between the as-constructed model (green) and the current profile (red), which may provide an indication of the presence of growth on the floor of the channel or movement in the overall concrete structure. 

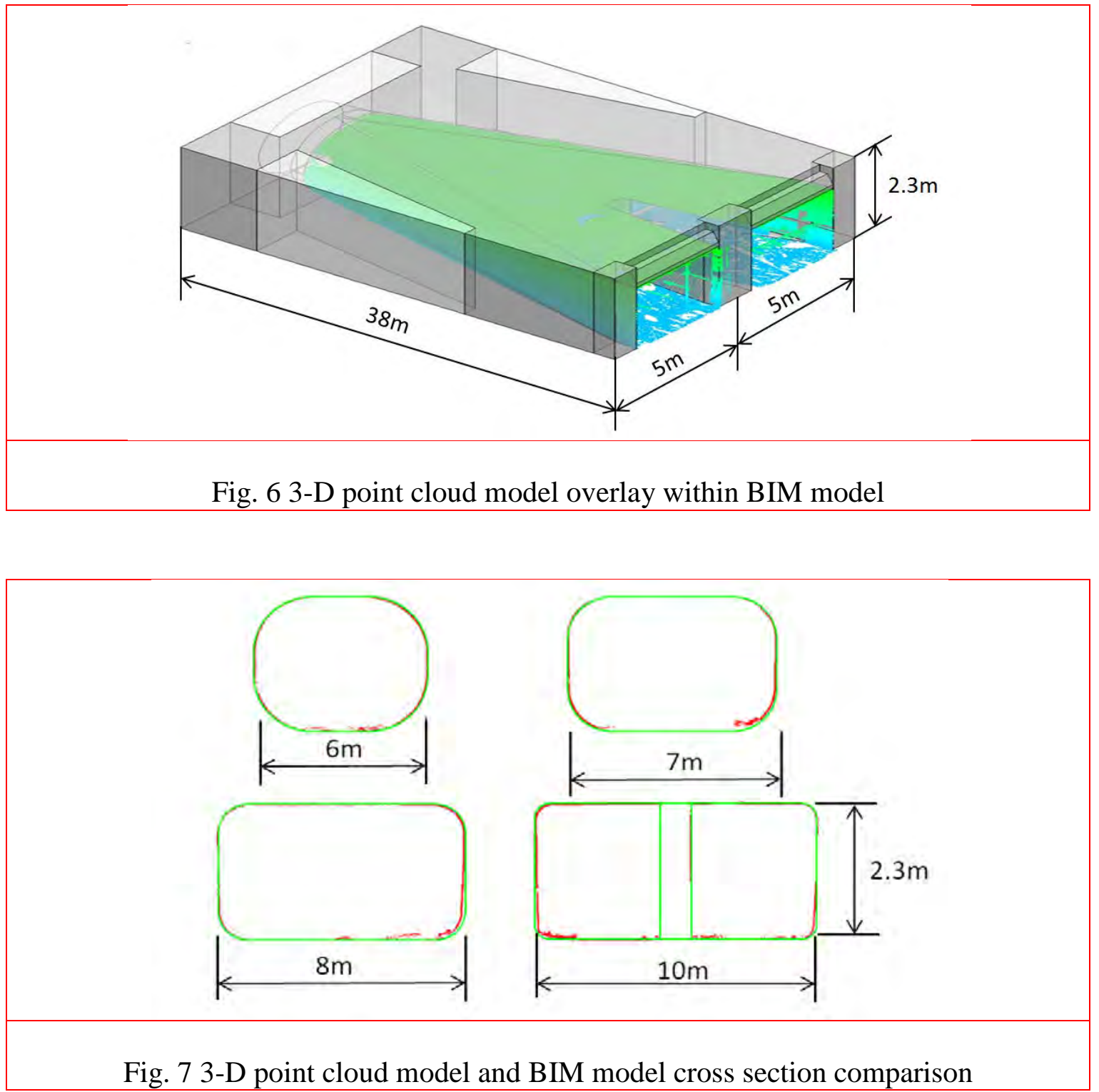

The key benefit of utilising BIM and laser scanned models is the ability to effectively monitor the rate of decay of a given structure. For bridges, this may be settlement at the abutments or batter, subsidence of the approaches or even deflection of a deck member. This additionally allows engineers to track the presence of changes in the surface profile, such as spalling to the concrete cover, vegetation growth of the bearing shelves, and subsidence at the toe of a batter protection. Through using 3-D laser scanning and comparing to an original BIM model, it is possible to track the decay of a structure to a reasonable degree of accuracy and create a true asset management model that would allow engineers and asset owners to make more effective decisions on remediation activities. As against 3-D laser scanning, the traditional measurement manner is to use a survey instrument (the Trimble Robotic Total Station) that has an electronic theodolite integrated with an electronic distance meter. To analyse the target surface, inspectors need to survey a large number of points and document the coordinates of each point, which as a result, could accrue various types of measurement error. Besides, such an approach has been plagued with several limitations for instance, its accuracy depends on the volume of data sets; the electronic theodolite needs strict levelling and alignment before use; the glass corner cube prism reflectors (measuring sensors) could not reach an accuracy of less than $1.5 \mathrm{~mm}$, etc. In this project, each survey required one 
people to operate the Trimble TX8 laser scanner, one to set the target and one to adjust the target through observing the total station. Although this approach could not reduce the number of people required on the site, it minimised the impact of human actions on accuracy. It took circa 30 minutes for every scan (high resolution) and 2-3 hours to process the scan data. Overall, the 3-D laser scanning technology could improve more than $50 \%$ efficiency and save up to $60 \%$ of cost as against the traditional approach in surveying large-scale geographical environments. There are several survey providers (contractors) who provide hardware (laser scanner), software (point clouds processing software) and service (personnel, feature-extraction services, mapping-grade accuracy, final needed output, etc.). The advantage of this option, which the client resorted to in this project, is that the client would not be burdened with training, data post-processing, technological obsolescence of equipment, and equipment maintenance.

\section{Discussion of identified challenges for integration into current practice}

\subsection{Uniform governance over BIM and advanced imaging technology}

While the research presented proposes an integrated system that noticeably addresses a wide range of issues that stem from the rigidity and minimalism of current systems, there exist hindrances to the adoption of a BIM and keypoint-integrated approach in bridge asset management. The hurdles are not perpetuated by the limitations of the current technology, but rather the regulation of governing authorities. It has been widely questioned whether current paper-based inspection processes are still providing the answers to the complex problems of modern infrastructure (Kamya 2010). The UK Government has led the way, establishing a BIM Task Group with the sole aim of defining a progressive implementation strategy for BIM and reducing cited barriers. However, within Australia there still remains no clear direction or governance provided and hence, provides no incentive for asset owners to take the leap towards more intelligent systems and processes.

\subsection{Modelling existing infrastructure}

BIM has been proven to be a powerful process that can produce direct savings for largescale bridge structures that have been in service for a number of years. However, the key challenge that appears to be limiting implementation in bridge operations is the ability to affordably and efficiently produce BIM models for all existing bridges that are not necessarily large scale, nor as important to the road network as that of the Pyrmont Bridge. Traditionally, there is limited data, albeit as-constructed information, that is available for producing a model of the structure. When taking this in conjunction with the considerable time required to produce a BIM model of a bridge, this only appears to compound the problem.

While there are techniques such as photogrammetric modelling and point-cloud surveys that may be conducted to produce a model for a given structure, this may not be suitable for the case of asset owners with tens of thousands of bridges/culverts within their inventory. Although this does not preclude the use of BIM for asset management, without a resolute approach that will be suitable for collecting and managing all assets using BIM, the reservation to complete adoption of the new technology will likely remain. 


\subsection{Aligning BIM at a corporate level}

Another key challenge to the BIM-oriented future is the ability to find common ground in terms of modelling software. For the design and detailing phase, there remain numerous suites and packages on the market that are capable of producing a full detailed design model. As is the case for all decisions made at a corporate level over software adoption, there is not one size that fits all where a package will suit every organisation. While a BIM model may be exported to a 'platform-neutral' IFC model for file exchange with different suites, it is still not a perfect solution to address information transfer constraints. Hence the challenge is to find a mutual approach/software that will not restrict governments and hence, organisations to a single platform, i.e. TEKLA, Bentley and Autodesk (Anderson et al. 2012).

\section{Overcoming the current constraints}

Ultimately, what is required is a commitment from the government, local authorities and other key stakeholders towards developing a roadmap or implementation strategy for BIM in asset management, where there are clearly defined indicators and timeframes. However, to achieve this, as-built models of all structures would likely need to be developed for all assets within the inventory of key asset owners, such as the Queensland Department of Transport and Main Roads, the Roads Corporation of Victoria and the Australian Rail Track Corporation. While BIM and advanced imaging technology may be adopted on a project-byproject basis, the efficiency and cost-effectiveness will be lost without the widespread adoption of a more proactive approach to maintenance. Taking lessons learnt from the Crossrail project and UK's BIM Industry Working Group, this should ideally be undertaken by defining distinct BIM maturity stages, whereby BIM-integrated asset management should be achieved by means of progressive development.

While the immediate implementation of a BIM appears to be constrained by the wider governance, there is still significant savings to be afforded by BIM if the challenge of producing models for existing infrastructure can be addressed. One option is to develop 'notto-scale' BIM models for the existing bridges which are produced from a defined set of inputs. By using BIM software with an open API framework, it is possible to produce a script to generate a baseline bridge model from the defined list of inputs. While the model does not take into account any details that are unique to the bridge (such as skew, camber, spacing between elements) and is dimensionally constrained, it provides a quick representation of the overall structure that can be used on site and can be developed further. This would ultimately enable the structure to be managed using BIM and hence, time and cost savings to be afforded (Lillenstein 2015). While the use of a model that is not geometrically accurate will be constrained for structural assessments, it enables asset owners to exploit the benefits of visually representing the structure and storing information in a single-source of truth. As outlined, the benefit of BIM is that it is able to be queried, with raw information 'dumped' back to excel for use in the current databases. This would ultimately provide a starting point for BIM and would help to familiarise key stakeholders and asset owners with BIM, whilst filling the void between complete as-built models and those existing assets without. However, for full integration of the technology into current practice, it is necessary to assess methods for developing geometrically accurate models with detailed parametric information assigned to the model in addition to identifying systems that are best suited to the needs of the asset owner. However, the means of developing models for the full inventory of asset owners is largely dependent upon the type of bridge structures encountered and may be addressed 
through such means as laser scanning, photogrammetric modelling and deriving models from as-constructed drawings.

As highlighted, there remains the predicament of different operating platforms. While this is not necessarily something that will be completely addressed, there are continually steps being taken by software companies to find a middle ground, whether it be through reducing the data exchange issues of IFC or developing plugins to accept different model formats (Trimble Navigation Ltd 2015).

\section{Conclusion}

Maintenance and facility operations remain the most significant phase of a project lifecycle, ensuring the ongoing serviceability of a structure and safety to the travelling public and users. With limited research into optimising the current practices and introducing alternative approaches to collecting and integrating the asset management phase, it is clear that the asset management industry has stagnated and remained fixed on antiquated and obsolete practices. In this paper, the theory behind the development of advanced imaging processes and integration with BIM has been discussed. The use of advanced imaging techniques, in particular keypoint-based texture recognition, presents the potential to create a consistent means of inspecting structures by processing images collected from the visual inspection and housed in the BIM-asset management model. Besides, for quality assurance that is important in assets performance management, BIM and advanced imaging integrated techniques are envisaged to be of great help in streamlining and coping with the onerous and high-risk field work. The following are the conclusions drawn from the studies conducted:

1) The ability to create a single point of truth and document the deterioration and works undertaken for a structure can significantly influence the evaluation of a structure's current condition and the need for further remediation.

2) Advanced imaging techniques have the potential to create a consistent approach to inspecting a structure and assessing for visual signs of deterioration. However, for this to be realised, further development of the machine learning algorithm and BIM interface are required.

3) BIM has illustrated the ability to ensure collaboration and knowledge transfer across the entire lifecycle of a project, enabling more efficient and informed decisions throughout the asset management phase.

4) For the implementation of BIM in bridge asset management, greater governance and steps towards standardisation, methodology for model generation and cross-platform collaboration issues are required to be addressed.

\section{Acknowledgement}

The authors wish to thank Shanghai Investigation Design \& Research Institute Co., Ltd in China for providing the case study of the Yongxin Floodgate Project.

The authors would like to additionally acknowledge the information provided by the Sydney Harbour Foreshore Authority for the Pyrmont Bridge BrIM project. 


\section{References}

Adhikari, R.S., Moselhi, O., \& Bagchi, A. (2014). Image-based retrieval of concrete crack properties for bridge inspection. Automation in construction, 39, 180-194.

AEC Magazine (2014), 30 of the best mobile apps for BIM. [Online] Available at:http://aecmag.com/technology-mainmenu-35/678-mobile-apps-for-bim-professionals [Accessed 31March 2016].

Alampalli, S., \& Rehm, K.C. (2011), Impact of I-35W bridge failure on state transportation agency bridge inspection and evaluation programs,Paper presented at the 2011 Structures Congress, Florida, USA.

Anderson, A., Marsters, A., Sturts., Dossick, C., \& Neff, G. (2012). Construction to Operations Exchange: Challenges of Implementing COBie and BIM in a Large Owner Organisation. Construction Research Congress. 688-697.

Bu, G., Lee, J.H., Guan, H., Blumenstein, M., \& Loo, Y.C. (2012). Development of an integrated method for probabilistic bridge-deterioration modelling. Journal of Performance of Constructed Facilities, 28(2), 330-340.

Bu, G., Lee, J.H., Guan, H., Loo, Y.C., \& Blumenstein, M. (2014a). Implementation of Elman neural networks for enhancing reliability of integrated bridge deterioration model. Australian Journal of Structural Engineering, 15(1), 51-63.

Bu, G., Lee, J.H., Guan, H., Loo, Y.C., \& Blumenstein, M. (2014b). Prediction of long-term bridge performance: Integrated deterioration approach with case studies. Journal of Performance of Constructed Facilities, 29(3), 04014089.

Bu, G., Chanda, S., Guan, H., Jo, J., Blumenstein, M., \& Loo, Y.C. (2015). Crack detection using a texture analysis-based technique for visual bridge inspection. Electronic Journal of Structural Engineering, 14(1), 41-48.

Huang, H., Guo, W., \& Zhang, Y. (2008). Detection of copy-move forgery in digital images using SIFT algorithm. Paper presented at the Computational Intelligence and Industrial Application, 2008. PACIIA'08. Pacific-Asia Workshop on.

Chan, B., Guan, H., Jo, J., \& Blumenstein, M. (2015). Towards UAV-Based bridge inspection systems: a review and an application perspective, Structural Monitoring and Maintenance, 2(3), 283-300.

Crossrail Ltd (2015), Crossrail and Bentley Systems launch UK's first dedicated building information modelling academy. [Online] Available at: http://www.crossrail.co.uk/news/articles/crossrail-bentley-systems-launch-uks-firstdedicated-building-information-modelling-academy [Accessed 8 November 2015].

Department of Transport and Main Roads. (2004), Bridge inspection manual, Queensland Government, Queensland, Australia.

DiBernardo, S. (2012), Integrated modelling system for bridge asset management - case study,Paper presented at the 2012 Structures Congress, Florida, USA.

Figueiredo, E., Park, G., Farrar, C.R., Worden, K., \& Figueiras, J. (2011). Machine learning algorithms for damage detection under operational and environmental variability. Structural Health Monitoring, 10(6), 559-572.

BIM industry working group. (2011), A report for the Government Construction Client Group Building Information Modelling (BIM) Working Party Strategy Paper, available on-line at http://www.bimtaskgroup.org/wp-content/uploads/2012/03/BISBIM-strategyReport.pdf (accessed March 2016).

Hardin, B., \& McCool, D. (2015). BIM and construction management: proven tools, methods, and workflows: John Wiley \& Sons.

Jacobi, J. (2011). 4D BIM or Simulation-Based Modelling. Structure Magazine, 17-18. 
Kamya, B. (2010), Bridge inspection; Are we getting it right? Bridge Maintenance, Safety,

\section{Kingdom.}

Lee, J.H., Guan, H., Loo, Y.C., Blumenstein, M., \& Wang, X.P. (2011). Modelling long-term bridge deterioration at a structural member level using artificial intelligence techniques, Applied Mechanics and Materials, Volume 99-100, 444-453.

Lillenstein, J. (2015), “Pyrmont Bridge enters the 3D world”, Engineers Australia Magazine, July.

Marzouk, M., \& Abdelaty, A. (2012). Maintaining subways infrastructures using BIM. Paper presented at the Proceedings of Construction Research Congress. 2320-2328.

Marzouk, M.M., \& Hisham, M. (2011). Bridge information modelling in sustainable bridgemanagement. Proc. ICSDC, 457-466.

McGuire, B., Atadero, R., Clevenger, C., \& Ozbek, M. (2016). Bridge Information Modelling for Inspection and Evaluation, Journal of Bridge Engineering, 21(4), 05015076-1 04015076-9.

M-Six (2015). VEO Presentation - Pymont Bridge, Presentation, Newcastle.

Liu, R., \& Issa, R.R.A. (2016). Survey: Common Knowledge in BIM for Facility Management. Journal of Performance of Constructed Facilities, 30(3), 04015033-1 04015033-8.

Sahlman, W. (2015), Best Practice Asset Management BIM - Pyrmont Bridge Case Study, Sydney Harbour Foreshore Authority, Sydney, Australia.

Subhani, M., Li, J., Samali, B., \& Yan, N. (2013). Determination of the embedded lengths of electrical timber poles utilizing flexural wave generated from impacts, Australian Journal of Structural Engineering, 14(1), 85-96.

Takken, R. (2016). Bentley's Year In Infrastructure. GeoInformatics, 19(1), 28.

Taylor, M. (2013), "Moving London Forward - Crossrail: A Case Study in BIM”, Lake Constance 5D-Conference 2013, Konstanz, Germany, October.

Trimble Navigation Ltd (2015), Trimble Improves Construction Project Workflow with Tekla $\begin{array}{llll}\text { Structures } & 21 & \text { BIM Software. [Online] Available at: }\end{array}$ http://www.trimble.com/news/release.aspx?id=031215b [Accessed 8 November 2015].

Trimble Navigation Ltd (2016), The Connected Construction Site: Accurate Layout Where And When You Need It. [Online] Available at:http://www.trimble.com/construction/building-construction/RTS-Series-Robotic-TotalStations.aspx [Accessed 31March 2016].

Wang, Y., \& Hao, H. (2010). Integrated Health Monitoring for Reinforced Concrete Beams: An Experimental Study. $6^{\text {th }}$ Australasian Congress on Applied Mechanics, 1-10.

Wardhana, K. \& Hadipriono, F.C. (2003). Analysis of Recent Bridge Failures in the United States. Journal of Performance of Constructed Facilities, 17(3), 144-150. 